This Person Does Exist is an artistic approach to exploring a large dataset of photographic portraits in a randomised manner. The dataset was originally created by Nvidia Research Lab, which has scraped and analysed creative commons images from the popular image hosting platform Flickr. These pictures were then used to train a machine learning model which can create new stochastic images of faces.
w In contrast to a popular website that showcases the computer generated images, I am displaying random faces from the dataset with their corresponding metadata.

This essay looks into extractivist mechanisms in current machine learning techniques, using the internet to populate and refine databases, while focusing on artistic approaches that expose them I make the case for Dataset Art as an emerging field which reframes scientific corpora by placing them into galleries and exhibiting them as found objects online.

Finally, I argue that this artistic practice is a legitimate way of opening up a larger public discourse, although artists working with human data must be aware of ethical issues and responsibilities regarding privacy and consent.

\title{
This Person Does Exist
}

This practice has been widely unknown to the general public, as shown by interviews of affected individuals by the New York Times (Hill and Krolik 2019) and NBC News (Solon 2019).

Training sets were not made for human consumption, but rather created for

which affected black women in particular (Buolamwini and Gebru 2018), the the statistical analysis and modelling of patterns between individual dat Yeace Yahoo dataset. They called it Diversity in Faces (DiF) (Merler et al. 2019) and points. By shifting our gaze onto these vast collections, we can find hidden of individual efforts, from taking pictures to labelling and categorising them. In this paper, ldiscuss my approach of looking into one specific dat
works of art that have dealt with similar issues in the recent past. measurements with the aim of making face detection fairer. ${ }^{3}$ tive model, is more than 10 times smaller, as it only contains 70,000 images. INTRODUCTION TO FLICKR FACES tions for use and download are very clear, making it manageable in terms In 2018 the research lab of Nvidia, one of the leading companies for visual of size, license and ease-of-use to discover the underlying characteristics. square ratio with a dimension of $1024^{2} \mathrm{px}$ with the open-source library dlib. The library provides fast facial recognition and identifies 68 points around called Sty, published a paper introducing a machine leanning architecture the face outlining the chin, eyebrows, eyes, nose and mouth. The resulting tive adversarial networks (Goodfellow et al. 2014) in such a way that it was the dataset was finally checked by Amazon Mechanical Turk workers" to remove

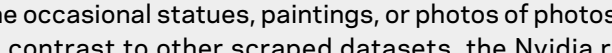

terms, scientists are able to abstract large amounts of images with a mode

other scraped datasets, the Nvidia researchers provide a that in turn outputs similar-looking pictures. In this case, they were able to $P$ generate realistic-looking images of human faces. As previous face datasets $n$ Dotograph from the FFHQ training set. As an investigative article from NBC News (Solon 2019) suggests, most photographers on Flickr are oblivious of were too low in resolution, ${ }^{1}$ a new corpus was created by scraping images the fact hat they are part of the larger IBM DiF dataset and they are divided Whi Creative Commons, Public Domain or U.S. Government Works licenses that this is the case in the FFHQ corpus too, as the researchers did not get Flickr-Faces-HQ(FFHQ). consent before creating the collection. 
To get a sense of scale of the dataset, I compiled all face images into a grid, was in the spring of 2020 and I faced technical roadblocks such as down- reducing the size of each image to 16 by $16 \mathrm{px}$ (Fig. 3). This simple montage and aligned face imas tomy server and built a wobsite ${ }^{8}$ that displays the

faces from people that do exist.9.

pixel values. This suppresses outliers, but it allows us to see an overall trend of the dataset. The resulting composite image The Flickr Face (Fig. 4) migh ceveal a trend towards sming and ight-skinned people in the data set. This be able to measure biases in this image set. with the unknown person and evokes a feeling for the images that were us
to train the generative model. As Flickr is used mostly by hobbyists and speakers at conferences ors, one can find portraits of children and families, includes more variety than other face image sets in terms "of age, ethricity and image background, and also has much better coverage of accessories
such as eyeglasses, sun-glasses, hats, etc." (Karras, Laine, and Aila 2019) niques to make large image sets experienceable and critique the unsolicited
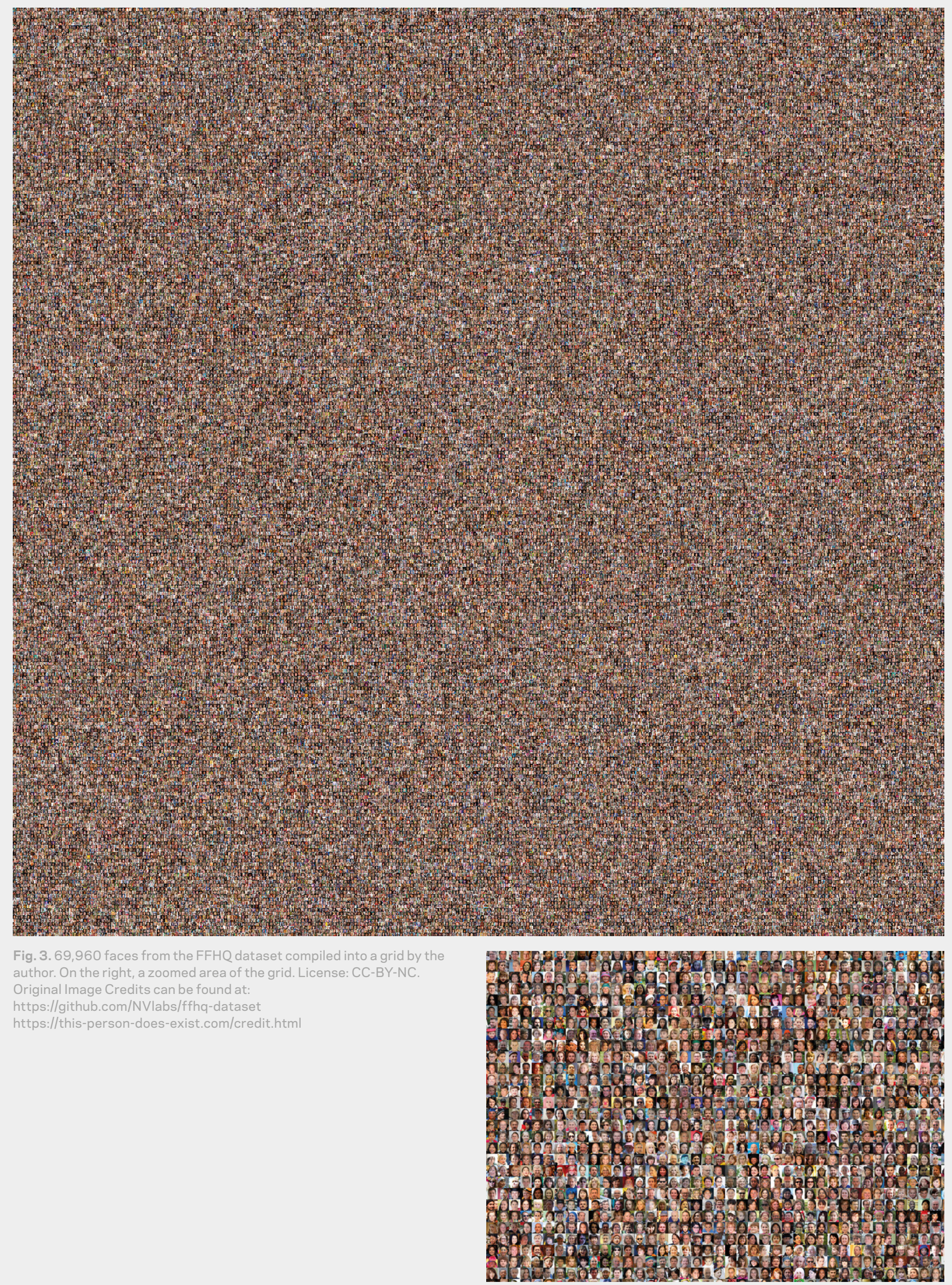
MEGAPIXELS, TRAINING HUMANS \& HUMANS OF AI

Since 2017 Adam Harvey and Jules LaPlace have been working on the art and research publication MegaPixels,,$^{10}$ where they analyse facial recognition and detection datasets. As aresult of their research and media attention, multiple
datasets have been revoked and removed, for example, the MegaFace dataset (Kemelmacher-Shlizerman et al. 2016) with roughly 4.7 million faces from Flickr (Thomee et al. 2016). The corpus was used by private companies for Furthermore, the dataset contains iuthors were not sufficiently credited collection, capturing and purchase of biometric information is prohibited (Hill and Krolik 2019).

Whil hing, they also found ways of making scraped ning much more personal.

2017 Adam Harvey showed ${ }^{11}$ an interactive installation for the Glass Room exhibition in which visitors' face landmarks are compared with images from

PICTORIAL

218
In 2019 the artist Trevor Paglen and researcher Kate Crawford collaborated
on an exhibibition titled Training Humans, dedicated to human artists focuse databases ${ }^{22}$
soft's COCO dataset (Lin et al. 2014), which was scraped from Flickr and Fig. 5). One of the main exhibits was a vast collection of human images from annotated by clickworkers for object recognition models.

the ImageNet dataset (Fig. 6), initiated by Stanford University's Al profesMechanical Turk workers. Some images of people fall into categories such as 'Bad Person, Call Girl, Drug Addict, Closet Queen, Convict' and so on (Crawford and Paglen 2019a). The artists used these absurd, racist an misogynistic labels to train ImageNet Roulte, a recognition algorithm that was action that followed was that 600,000 images were removed fhom ImageNet and, due to maintenance, ${ }^{14}$ the website and dataset have not been properly accessible since (Yang et al. 2019). But Training Humans comes with its own issues, as Michael J. Lyons (2020) recently wrote in a crilique of he work of Crawford and Paglen. Exhibinon-commercial scientific research. He criticises the double standards of Crawford and Paglen using human data without prior informed consent. Knowing fully about the ethical dilemma, the artists argue against privacy "to have the real conversation about what's going on with these $[A \mid$ systems" (Craver a images from the exhibition, it was sometimes too late for them. This was the
case of the photographed volunteers from the exhibited JAFFE dataset by Lyons, Kamachi, and Gyoba (1998), which was never intended for use as a "training set." Only after the exhibition closed did they realise that the images intended for scientific use were distributed all over the inter
ment and a bad example for emotion recognition research.
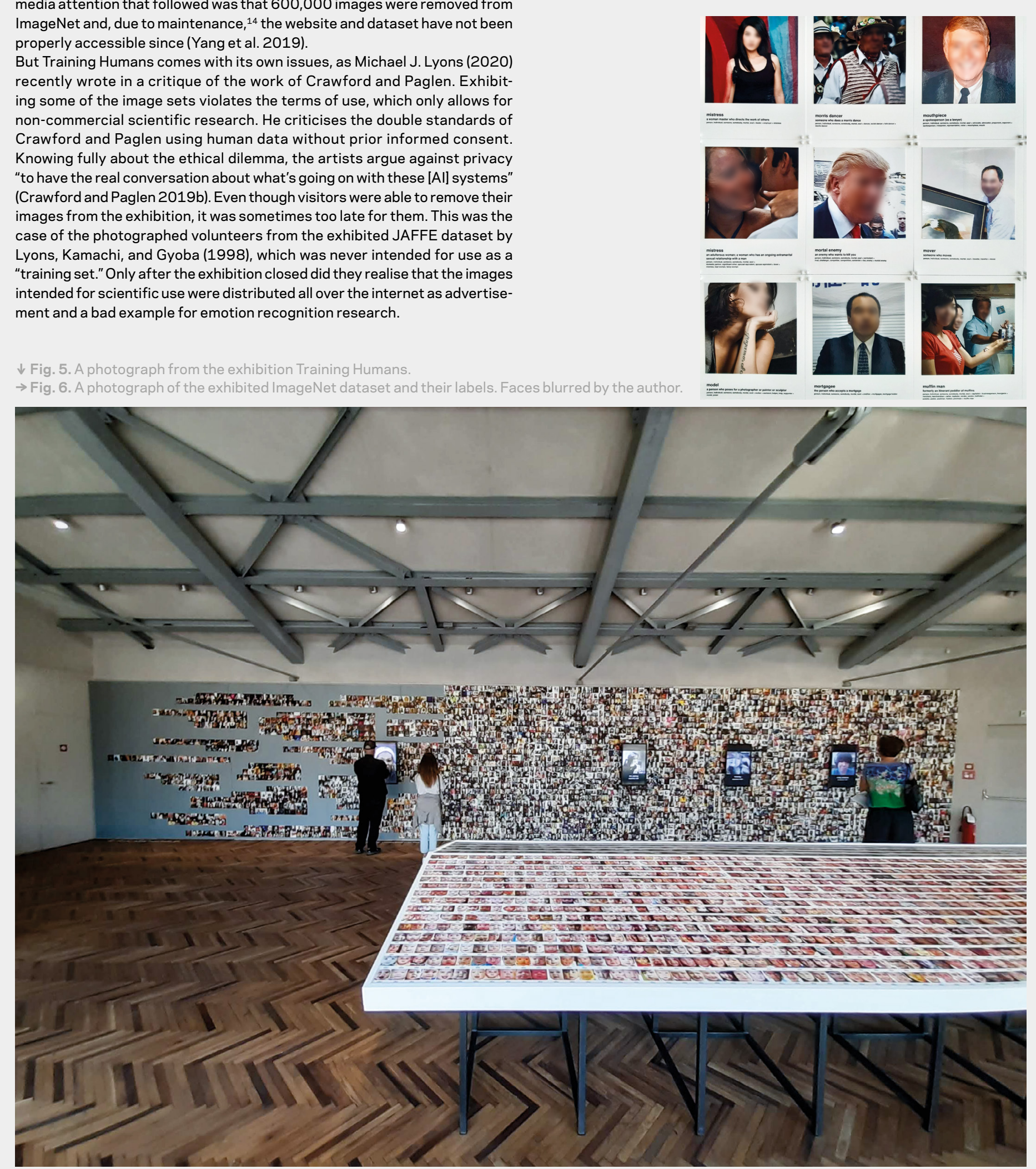
Matthias Schäfer

Matthias Schäfer is an artist who explores algorithmic cultures. As pitscher, exhibitions and festivals.

chäfer is currently enrolled in the Interface Cultures Master programme

of machine learning, automation and its core mechanism of data collecting.

\section{ENDNOTES}

Noticing the missing image attributions, the artist Philipp Schmitt published Artists have responded to the creation of these datasets by analysing and Common Objects in Context). Using YOLO (Redmon and Farhadi 2018), an attention and scientific discourse for institutions to remove questionable

er (Fig. 8), which superimposes images from the dataset onto another picture. With my research into the FFHQ dataset, I found common issues that tie it
. Finally, he produced a slideshow that fades between all images from the to the other presented works, which include the use of human data without Another artist, Sebastian Schmieg, has acknowledged the work of the un- Flickr and the use of clickworkers to refine the dataset. Other issues were Known clickworkers outlining objects for the $\mathrm{COCO}$ set by reating segmen- not present: the authors attributed the creators accordingly, created a tool nd drawn outlines (Fig. 9).

CONCLUSION

a CC non-commercial license itself.
Still, looking through the automatically cropped images of human faces (1) statistical occurrences. My work shows the person that does exist behind the Al model, but it does violate personality rights as much as the original dataset. This ethical dilemma needs to be explored further, but so far I think create awareness of practices in ML research.17

adversarial networks and detection algorithms, the exploits open licenses to do so. In most cases, the cort

1. For the preceding Paper' 'Progressive Growing of GANs for Improved Qualty, Stability, and Variation' (Karras et al. 2017) the authors up scaled images from the popular Ce
to meet their demands.

2. See https://github.com/NVlabs/ffhq-dataset

3. The inclusion of computer-generated face measurements, age and gender assumptions were highly crititized, because of their roots in com/tensorflow/datasets/issues/299) the dataset is not availa

4. See http://dlib.net/

ory of the FFHQ dataset

(endnote 2). Accessed 25 January 2021.
6.See https://nvlabs.github.iolffhq-dataset/search/

7.See https://thispersondoesnotexist.com/
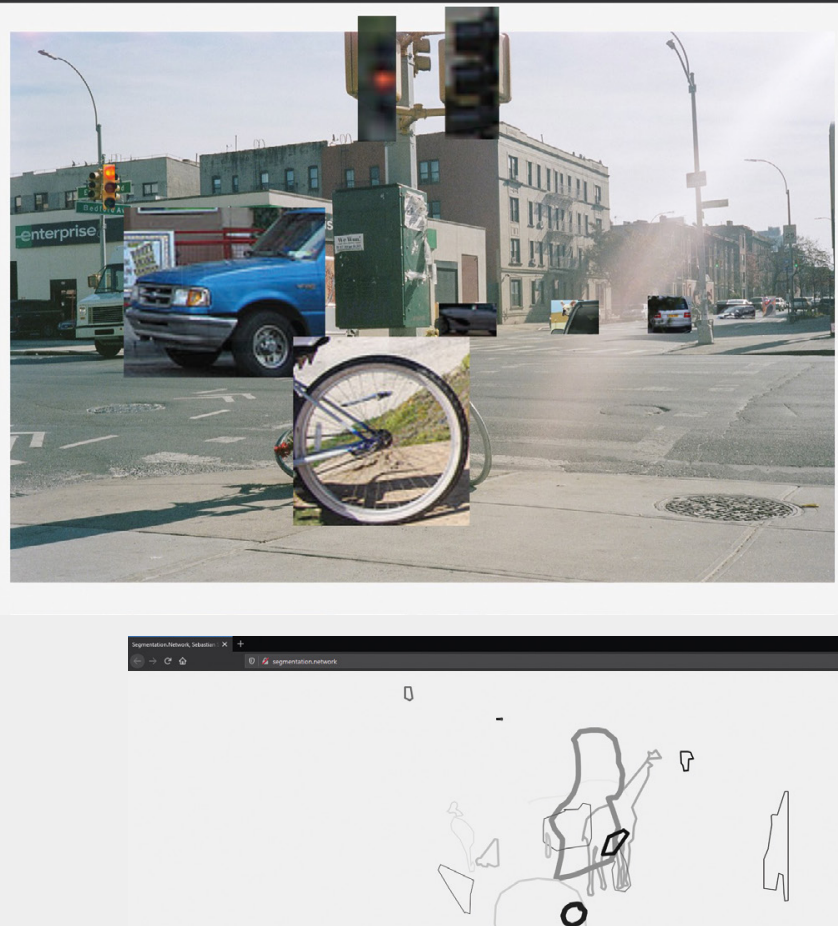
We way His works have been exhibited at transmediale Ars Electronice Festival, Xie Zilong photography museum, Roehrs \& Boetsch and multiple other

at the University of Art \& Design in Linz, where he is focusing on the field , Bing Xu, David 1014. "Generative Adversarial Networks". https://arxiv.org/abs/1406.2661

Surveillance Technology" New York Times, October 11. 2019

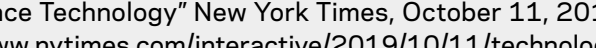
flickr-facial-recognition.htm

arras, Tero, Samuli Laine, and Timo Aila. 2019. "A Style-Based Generator (Cenerat Adversarial Networks."InIEEE/CVF Long Beach, California, 4396-4405.
https://doi.org/10.1109/CVPR.2019.00453

Karras, Tero, Timo Aila, Samuli Laine, and Jaakko Lehtinen. 2017."Progressive Growing of GANs for Improved Quality, Stability, and Variations, ICLR 2018, Vancouver, BC, Canada, April 30-May 3, 2018. https://arxiv.org/abs/1710.10196 ble from IBM, though it is still being publicised by IBM. the same concept, but the same concept, but that gets the
and crops them in the browser.
See http://thispersons

See http://thispersonexists.net/ and the corresponding tweet

10. See https://ahprojects.com/megapixels/ and https:/lexposing ai/

11. See MegaPixels: Faces, https:///ahprojects.com/megapixels-glassroom/ HUMANS", http://www.fondazioneprada.org/proj:ect/traininghumans/?lang=en

13. See Trevor Paglen, ImageNet Roulette

https://paglen.studio/2020/04/29/imagenet-roulette/ March 2021 the team updated https://image-net.org/. They ad-
dressed the criticism by removing 2702 synets and xpperion (cism by re 2702 synsets and experiment

15. See Philipp Schmitt, Humans of Al, https://humans-of.ail

16. See Sebastian Schmieg, Segmentation.Network http://segmentation.network/ further information on:
http://sebastianschmieg com /segmentation-network/

17. The publication http://plottingd.at/a/ explores datasets through artistic interfaces and critical reflections much further.

\section{REFERENCES}

Buolamwini, Joy, and Timnit Gebru. 2018. "Gender Shades: Intersectional Accuracy Disparities in Commercial Gender Classification." In Proceedings of the 1st Conference on Fairness, Accountability
and Transporency, PMLR 81, 77-91. Cambridge, Mass.: Proand Transparency, PMLR 81, 77-91. Cambridge, Mass.: Proceedings of Machine Learning Research. http:///proceedings.
mlr.press/v81/buolamwini18a/buolamwini18a.pdf

Crawford, Kate, and Trevor Paglen. 2019a. "Excavating Al". The Al Now Institute, NYU. Accessed January 24, 2021. https://www.excavating.ail

Crawford, Kate, and Trevor Paglen. 2019b. "What are the ethics of exhibiting
datasets of faces? An interview with Kate Crawford \& Trevor Paglen". Interview by Gaia Tedone. The Photographers' Gallery, https://www.youtube.com/watch?v=BSaYROvz29E

Deng, Jia, Wei Dong, Richard Socher, Li-Jia Li, Kai Li, and Li Fei-Fei. 2009.
"Imagenet: A large-scale hierarchical image database." In IEEE

M. SCHÄFER 


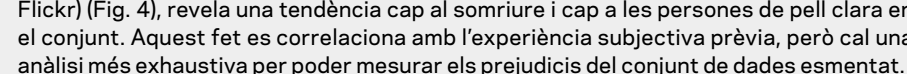

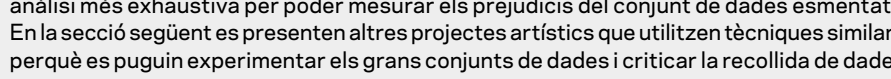

ELS PROJECTES IEXPOSICIONS MEGAPIXELS, "TRAINING HUMANS" I"HUMANS

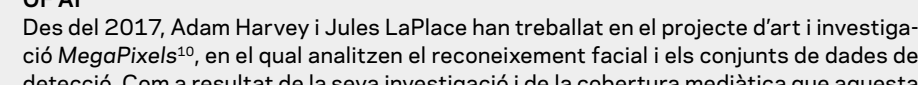

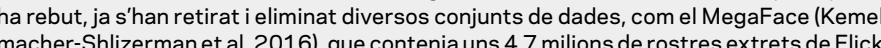

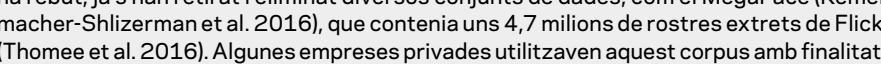

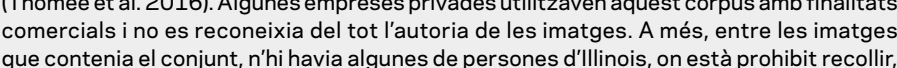

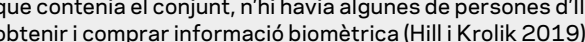

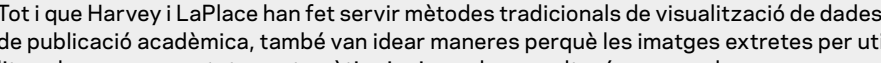

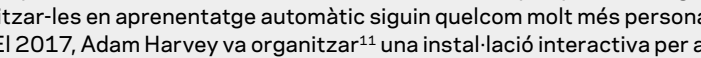

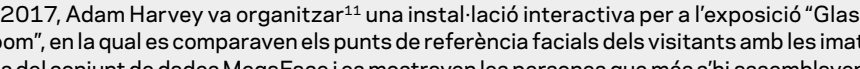

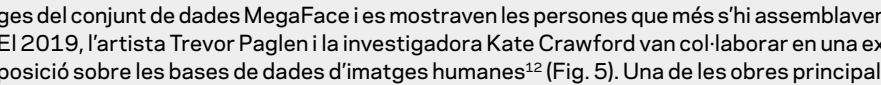

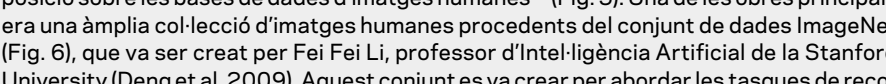

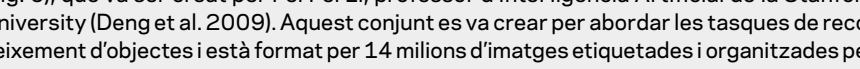

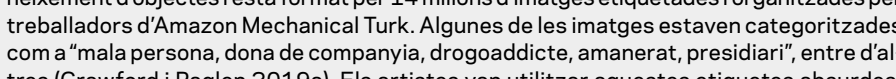

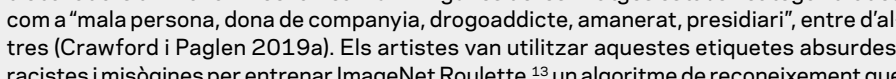

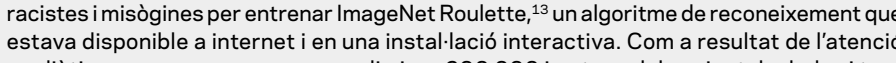

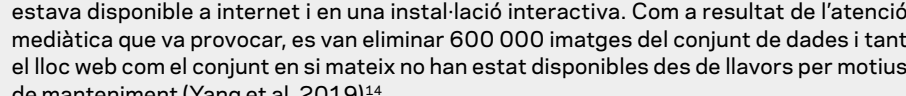

Traducció al Català

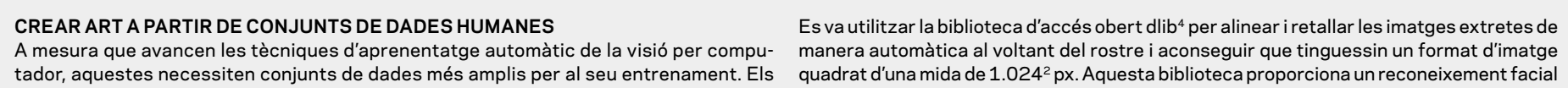

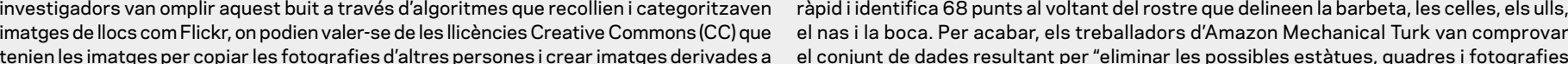

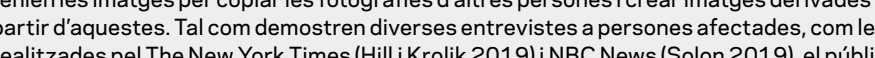

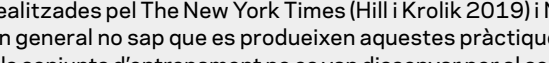

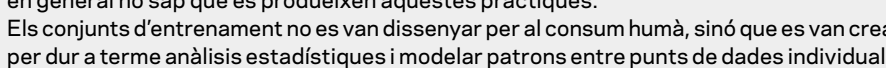

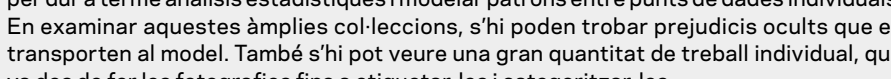

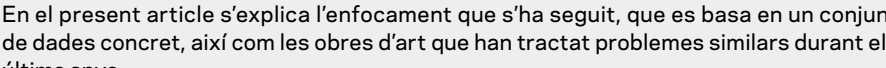

INTROOUCCIÓALSROSTRES DE FLCKR

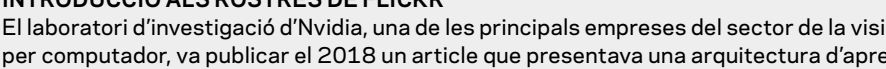

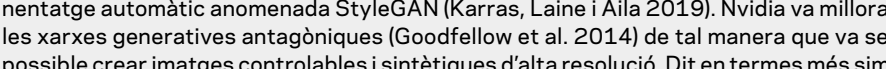

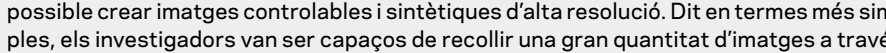

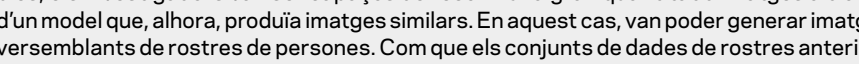

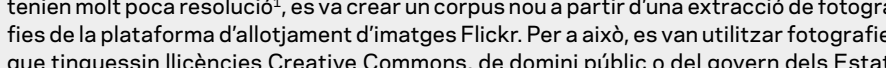

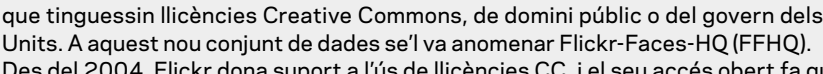

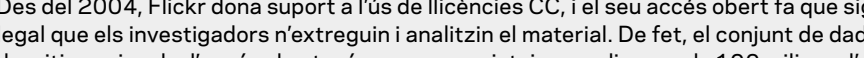

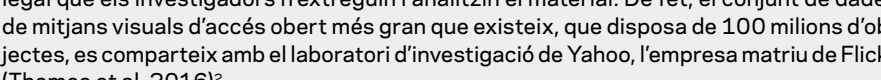

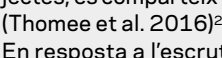

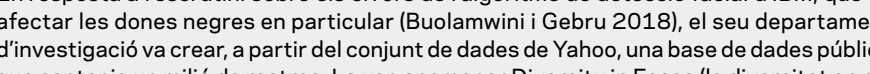

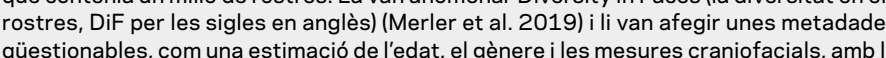

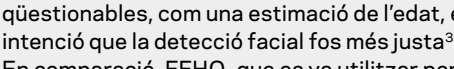

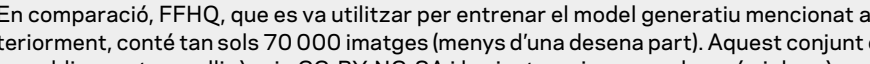

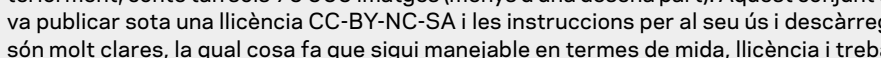

TEMES DE DISSENY \#37

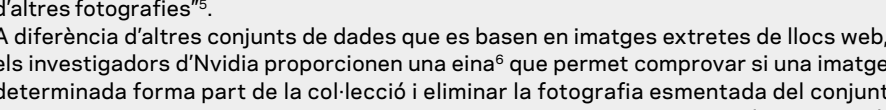

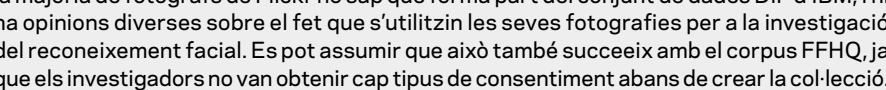
AQUESTA PERSONA Si QUE EXISTEIX

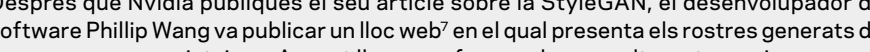

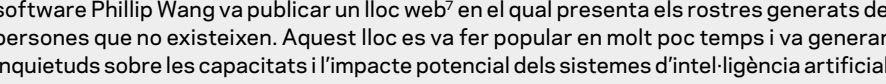

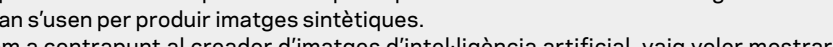

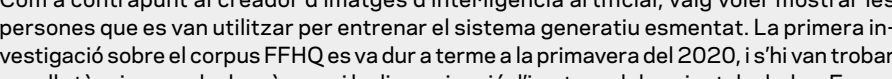

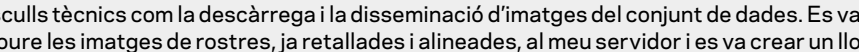

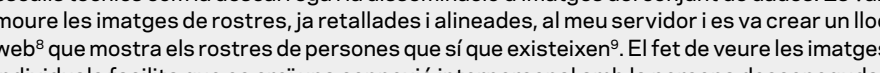

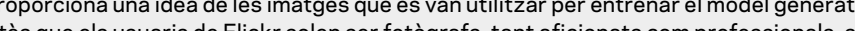

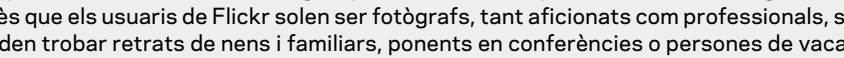

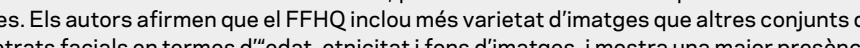

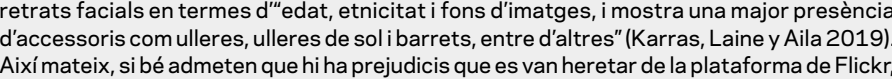

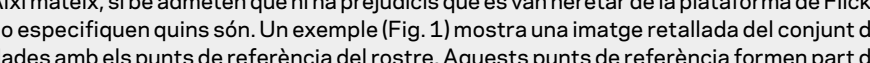

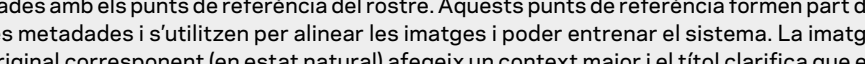

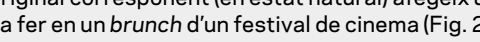

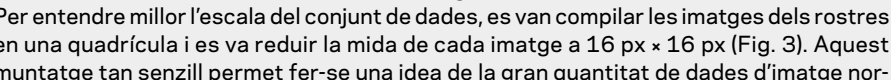
-

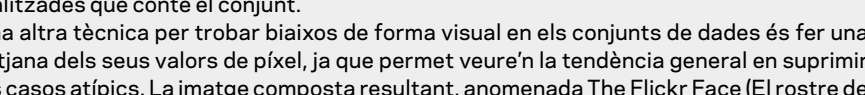
CÀPSULA IL'LUSTRADA 222

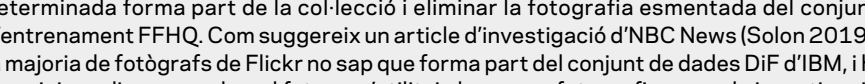

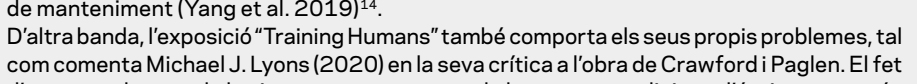

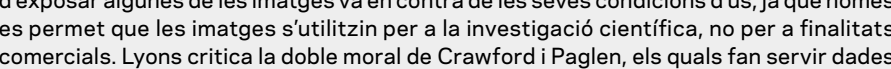
comercials. Lyons critica la doble moral de Crawford i Paglen, els suals fan servir dades
humanes sense havero obtingut un consentiment informat previ. Malgrat que coneixen (a)

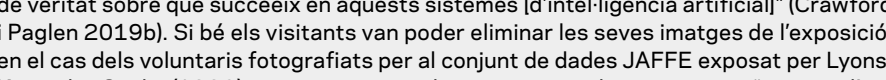
Kamachi G Gyoba (1998), que mai va estar destinat per a uttilitzar-se com a "conjunt d'e-

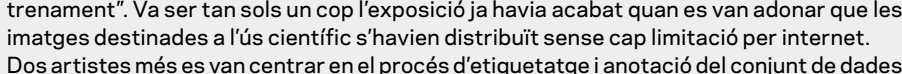

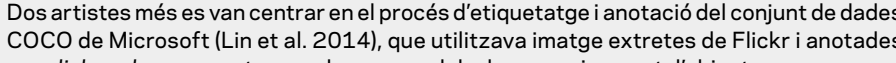
per clickworkers per entrenar els seus models de reconeixement d' 'objectes.
En adonar-se que no es reconeixial alautoria de les imatges, I'artista Philipp

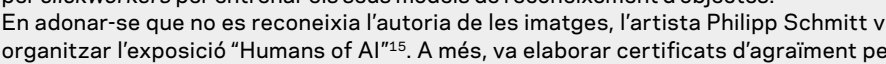

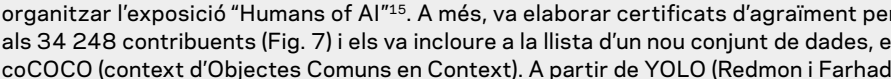

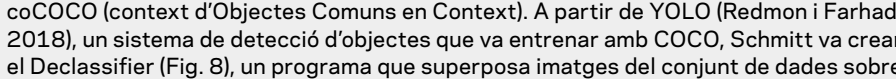

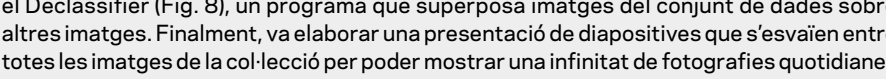
d'aficionats.
Un altre artista, Sebastian Schmieg, va reconèixer el treball dels clickworkers anònims

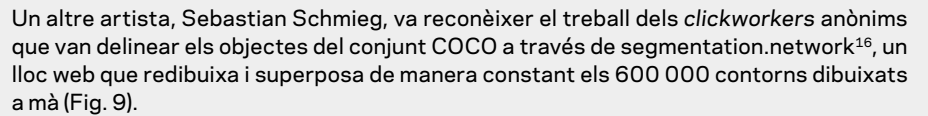
CONCLUSIONS
Sibée els srans sonjunts de dades han permès desenvolupar noves tecnologies de la aimatge
com les xarres generatives antagoniques i els algoritmes de detecció, la comunitat d'in-

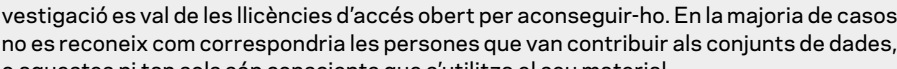

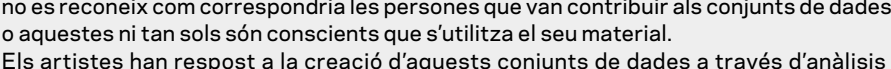
Els artistes han resposta a la creació d'aquests conjunts de dades a través d'anallisis
d'exposicions tant en galeries com online. Les seves obres fan que els grans conjunts de

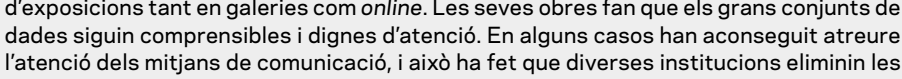

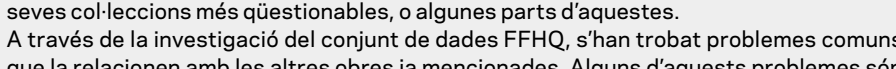
que la relacionen amb les altres obres ja mencionades. Alguns d'aquests problemes són
l'ús de les dades humanes sense tenir el consentiment informat previ, com les i matges

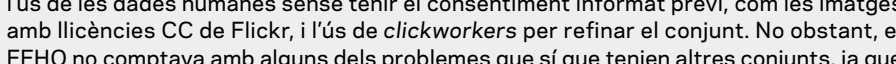

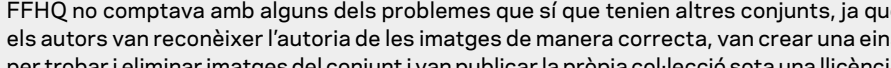

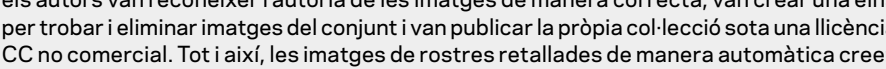

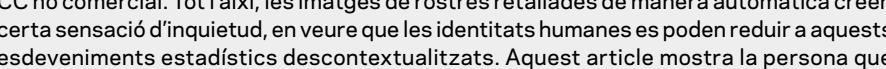
M. SCHÄFER
BIOGRAFIA

Matthias Schäfer
University of Art \& Design Linz

政

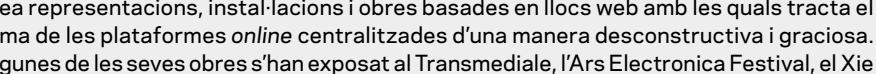

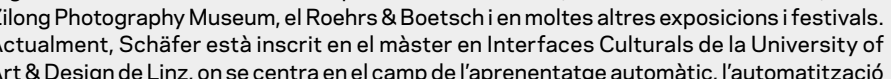
Art\& Design de Linz, on se centraenel camp de l'apter
el seu mecanisme central: la recollida de dades

NOTES FINALS

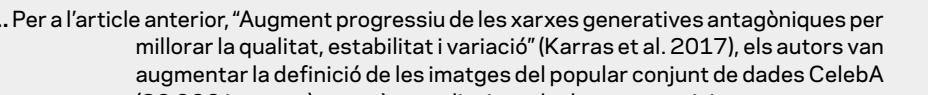

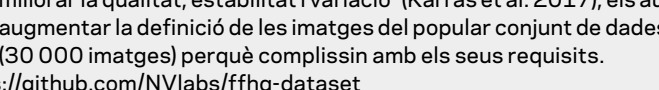

Hisuposicions sobre edadi igenere generades per ordinador

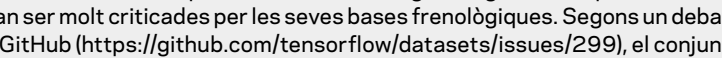

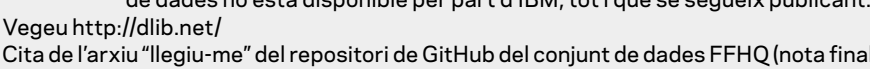

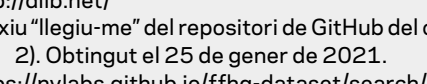

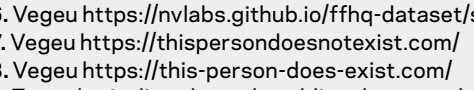

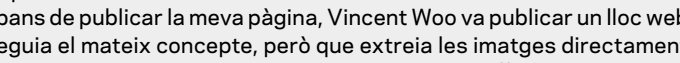

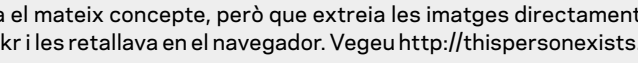
net/ie: tweet corresponent
https://twitter.com/fulligin/status/1335030372187312128

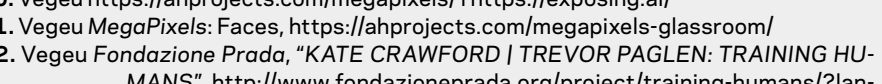
13. Vegeu Trevon
htrogs:en, ImageNent Roulette,
httogalen.studiol/2020/04

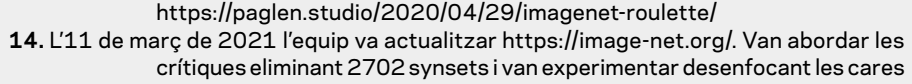

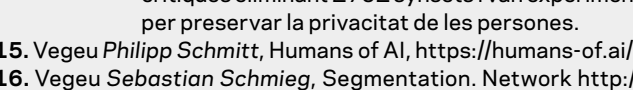

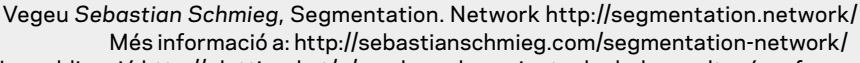

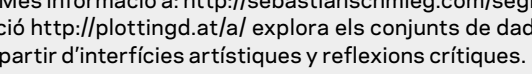

FIGURES

Fig. 1. Imatge retallada de $1.024 \mathrm{px} \times 1.024 \mathrm{px}$ del conjunt de dades $F F H Q$, amb els
punts de referència del rostre que provenen del mateix conjunt superposats per l'autor.
Fig. 2. Imatge enestat antural" extreta de Flickr, amb els punts de referència del ros-
tre del conjunt de dades superposades per l'autor. Llicència: CC-BY-2.O.

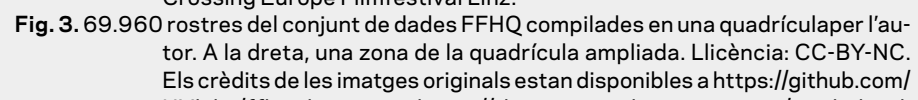

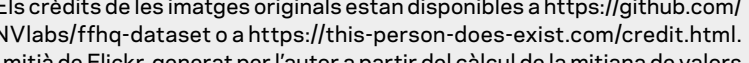

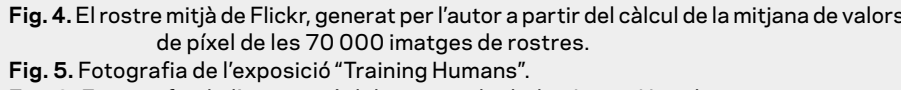

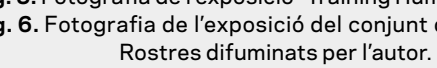

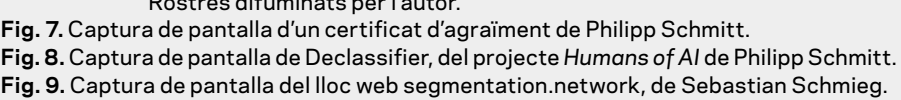
REFERĖNCIES 


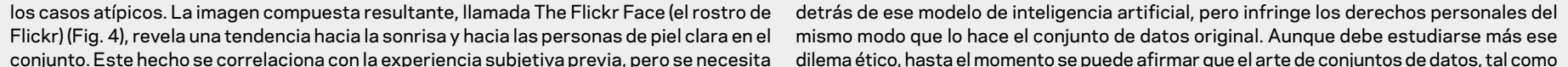

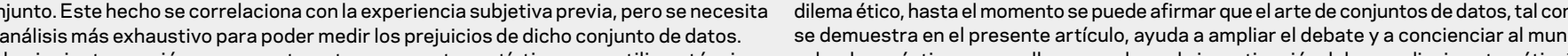
$\begin{array}{ll} & \end{array}$ (ocleccion de a datos humanos sin consentimiento previc

LOS PROYECTOS Y EXPOSICIONES MEGAPIXELS, "TRAIING HUMANS"Y

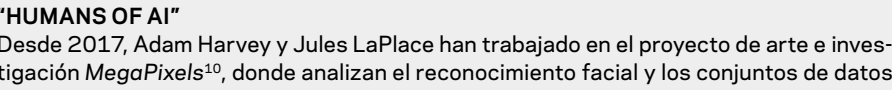
de detección. Como resultado de su investigación y de la cobertura medíatica aue esta

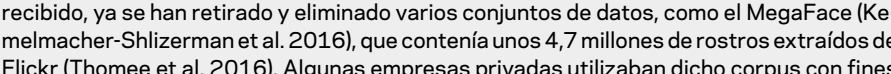
Flickr (Thomee et al. 2016). Algunas empresas privadas utilizaban dicho corpus con fines

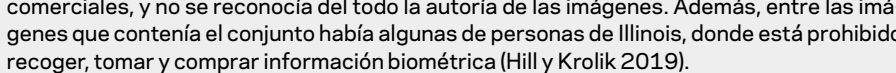

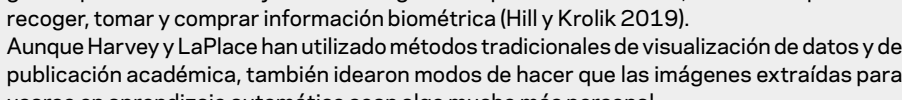
usarse en aprendizaje automaticos sean algo mucho más personn

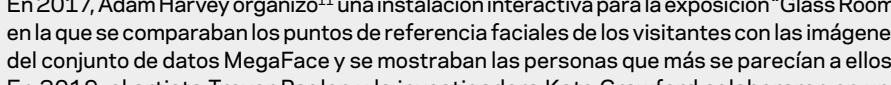
(2)

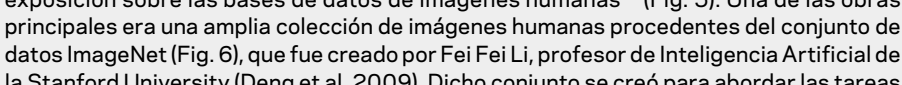

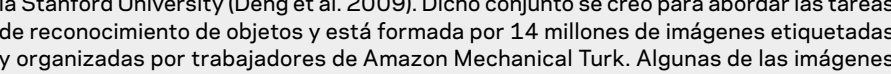

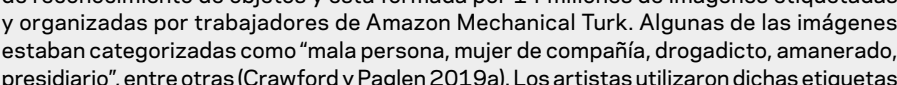

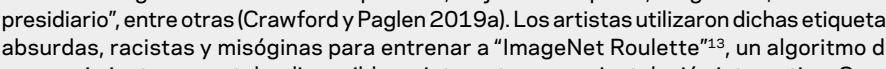

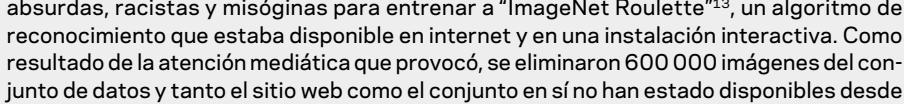
Sin embargo, la exposicición" Tranininiento(YYang et al. 2019)

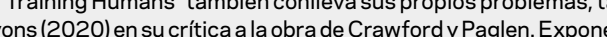

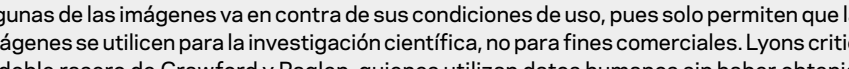
doble rasero de Crawford y Paglen, quienes utilizan datos humanos sin haber obtenid

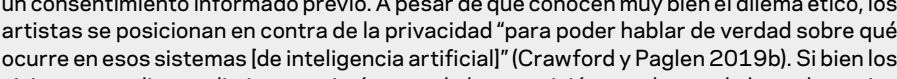

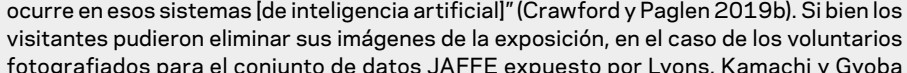

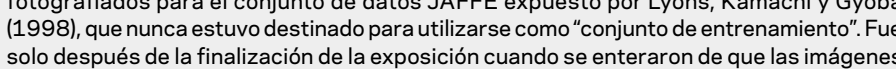
lestinadas al uso cienttfico se habian distribuido sin ninguna limitación por internet.

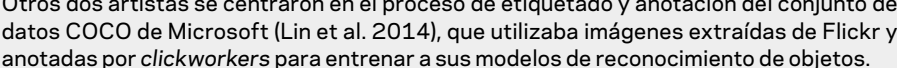

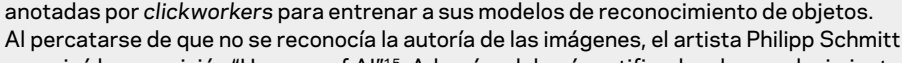

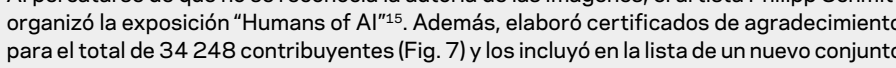

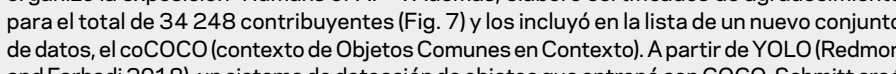

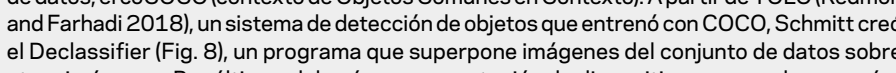

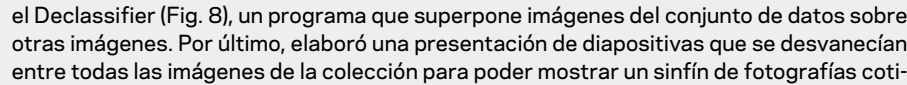

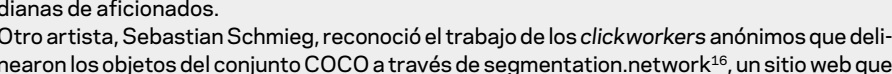

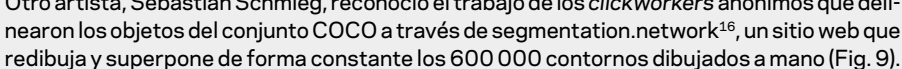

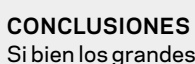
en los grandes conjuntos de datos han permitido desarrollar nuevas tecnologias de la

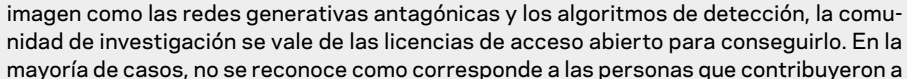
Mayoria de casos, no se reconoce como corresponde a las pers onas que contribuyeron
los conjuntos de datos, o estas ni siquiera son conscientes de que se utiliza su material. Los artistas hanr respondido a a creación de dichos conjuntos de datos através de análisisy

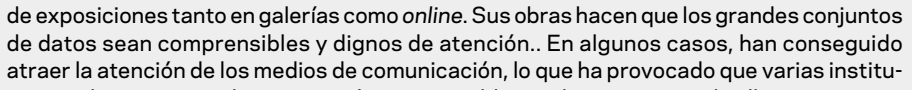

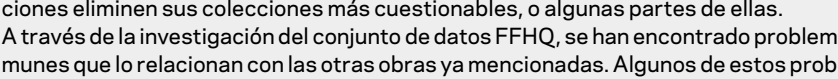

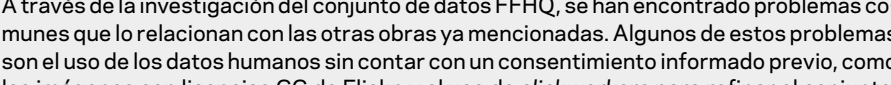

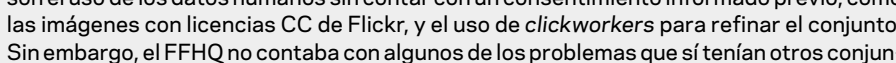

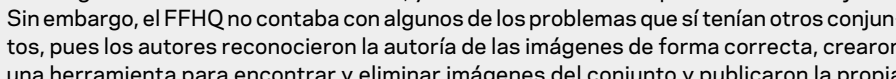

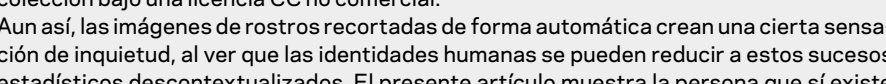
Matthias Schäfer
versity of Art \& Design Linz Matthias Schäfer es un artista que explora las culturas del los algoritmos. Desde Pitscher,
creareapresentaciones, intstalacionesy obrass basadas sen sitios web con los que tratael tema de las plataformas online centralizadas de un modo deconstructivo y gracioso. Algunas de sus obras se han expuesto en el transmediale, el Ars Electronica Festival, el Xie Zilong
photography museum, el Roehrs $\&$ Booetsch y en muchas otras exposiciones y festivales.

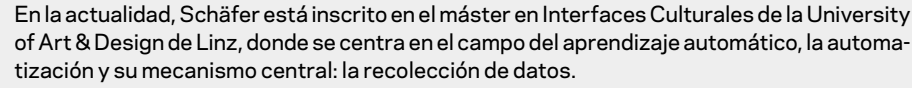

NOTAS FINALES

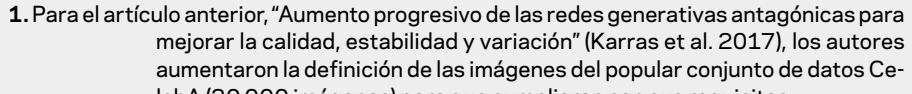

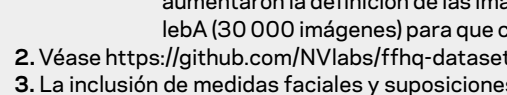

de medidas faciales y y suposiciciones sobre edad y género generadas por or-

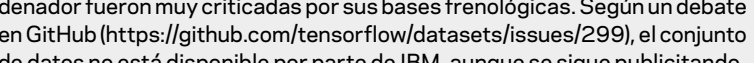

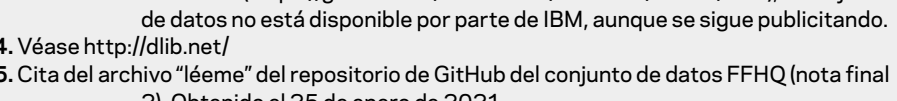
2l. Obtenido el 25 de enero de 2021 .
Véase https:/l/vilabs.github.olfffha-dataset/search/

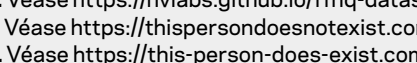

dias antes de publicar mi página web, Vicent Woo publicóo otra conel mismo

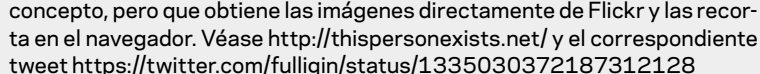

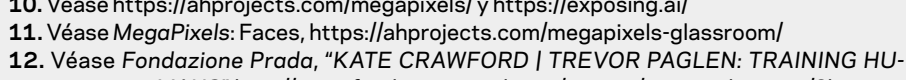
13. Véase Trevor Paglen, imageNet Roulette,

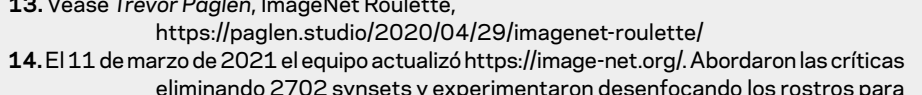
preservar la privacidad de las personas.
15. Véase

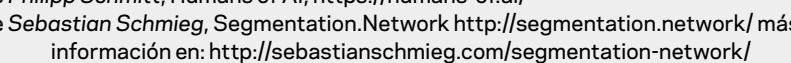

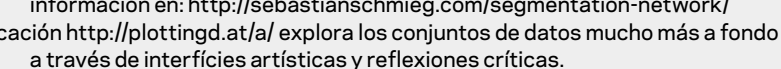
FIGURAS

Fig. 1. Imagen recortadade $1024 \mathrm{px} \times 1024 \mathrm{px}$ del conjunto de datos $\mathrm{FFHQ}$, con los puntos
de referencia del rostro que provienen del mismo conjunto superpuestos
porel autor

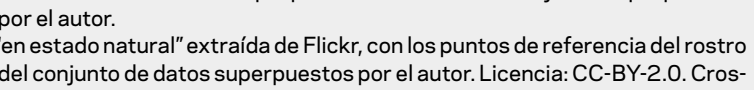
del conjunto de datos superpuestos por el autor. Licencia: CC-BY-2..O. Cros-
sing Europe F Filmfestival Linz.

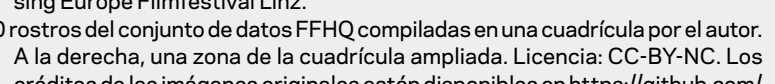

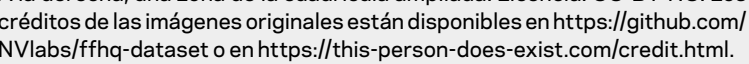
Fig. 4. El rostro medio de Flickr, generado por el autor a partir del cálculo de la media de
valores de pixel de las 70000 imágenes de rostros. valores de pixel de las 70000 imágenes de rostros
Fig. 5. Fotografia de la laxposición "Training Humans" difuminados por el a autor.

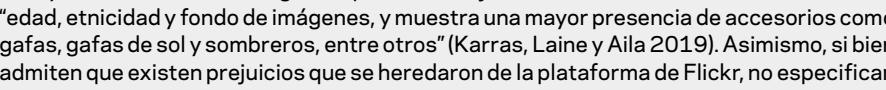
cuáles son. Un ejemplol(Fig. 1 ) muestra unaimagenrecortada del conjunto de datos con los

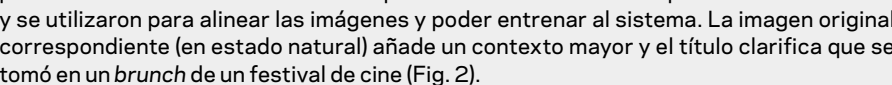
tomóen un brunch de un festival de cine (Fig. 2).
Para entender mejor la escala del conjunto de datos, se compilaron las imágenes de los

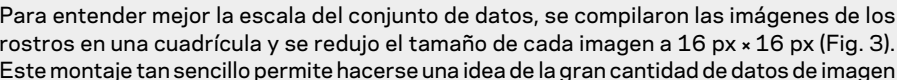

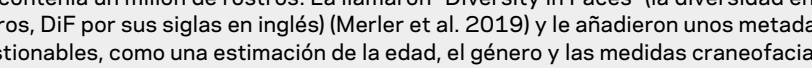

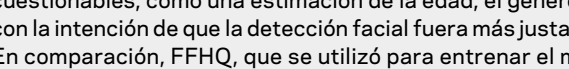

REFERENCIAS

Ver listado completo de referencias en la página 221 